Pacific Journal of Mathematics

CONCERNING NONNEGATIVE MATRICES AND DOUBLe 


\title{
CONCERNING NONNEGATIVE MATRICES AND DOUBLY STOCHASTIC MATRICES
}

\author{
RICHARD SINKHORN AND PAUL KNOPP
}

This paper is concerned with the condition for the convergence to a doubly stochastic limit of a sequence of matrices obtained from a nonnegative matrix $A$ by alternately scaling the rows and columns of $A$ and with the condition for the existence of diagonal matrices $D_{1}$ and $D_{2}$ with positive main diagonals such that $D_{1} A D_{2}$ is doubly stochastic.

The result is the following. The sequence of matrices converges to a doubly stochastic limit if and only if the matrix $A$ contains at least one positive diagonal. A necessary and sufficient condition that there exist diagonal matrices $D_{1}$ and $D_{2}$ with positive main diagonals such that $D_{1} A D_{2}$ is both doubly stochastic and the limit of the iteration is that $A \neq 0$ and each positive entry of $A$ is contained in a positive diagonal. The form $D_{1} A D_{2}$ is unique, and $D_{1}$ and $D_{2}$ are unique up to a positive scalar multiple if and only if $A$ is fully indecomposable.

Sinkhorn [6] has shown that corresponding to each positive square matrix $A$ there is a unique doubly stochastic matrix of the form $D_{1} A D_{2}$ where $D_{1}$ and $D_{2}$ are diagonal matrices with positive main diagonals. The matrices $D_{1}$ and $D_{2}$ are themselves unique up to a scalar factor. The matrix $D_{1} A D_{2}$ can be obtained as a limit of the sequence of matrices generated by alternately normalizing the rows and columns of $A$. But it was shown by example that for nonnegative matrices the iteration does not always converge, and even when it does, the $D_{1}$ and $D_{2}$ do not always exist.

Marcus and Newman [4] and Maxfield and Minc [5] gave some consideration to this problem for symmetric matrices.

In a recent communication with $\mathrm{H}$. Schneider, the authors learned that Brualdi, Parter and Schneider [2] have independently obtained some of the results of this paper by employing different techniques.

Definitions. If $A$ is an $N \times N$ matrix and $\sigma$ is a permutation of $\{1, \cdots, N\}$, then the sequence of elements $a_{1, \sigma(1)}, \cdots, a_{N, \sigma(N)}$ is called the diagonal of $A$ corresponding to $\sigma$. If $\sigma$ is the identity, the diagonal is called the main diagonal.

If $A$ is a nonnegative square matrix, $A$ is said to have total support if $A \neq 0$ and if every positive element of $A$ lies on a positive diagonal. A nonnegative matrix that contains a positive diagonal is said to have support.

The notation $A[\mu \mid \nu], A(\mu \mid \nu]$, etc. is that of [3, pp. 10-11]. 
THEOREM. Let $A$ be a nonnegative $N \times N$ matrix. A necessary and sufficient condition that there exist a doubly stochastic matrix $B$ of the form $D_{1} A D_{2}$ where $D_{1}$ and $D_{2}$ are diagonal matrices with positive main diagonals is that $A$ has total support. If $B$ exists then it is unique. Also $D_{1}$ and $D_{2}$ are unique up to a scalar multiple if and only if $A$ is fully indecomposable.

$A$ necessary and sufficient condition that the iterative process of alternately normalizing the rows and columns of $A$ will converge to a doubly stochastic limit is that A has support. If A has total support, this limit is the described matrix $D_{1} A D_{2}$. If $A$ has support which is not total, this limit cannot be of the form $D_{1} A D_{2}$.

Proof. We first demonstrate uniqueness. Suppose $B=D_{1} A D_{2}$ and $B^{\prime}=D_{1}^{\prime} A D_{2}^{\prime}$ are doubly stochastic where $D_{1}=\operatorname{diag}\left(x_{1}, \cdots, x_{N}\right), D_{2}=$ $\operatorname{diag}\left(y_{1}, \cdots, y_{N}\right), D_{1}^{\prime}=\operatorname{diag}\left(x_{1}^{\prime}, \cdots, x_{N}^{\prime}\right)$, and $D_{2}^{\prime}=\operatorname{diag}\left(y_{1}^{\prime}, \cdots, y_{N}^{\prime}\right)$. If $p_{i}=x_{i}^{\prime} / x_{i}, q_{j}=y_{j}^{\prime} / y_{j}$,

$$
\begin{aligned}
\sum_{i} x_{i} a_{i j} y_{j} & =1 ; & & \sum_{j} x_{i} a_{i j} y_{j}=1 \\
\sum_{i} p_{i} x_{i} a_{i j} q_{j} y_{j} & =1 ; & & \sum_{j} p_{i} x_{i} a_{i j} q_{j} y_{j}=1 .
\end{aligned}
$$

Let $E_{j}=\left\{i \mid a_{i j}>0\right\}, F_{i}=\left\{j \mid a_{i j}>0\right\}$ and put

$$
m=\left\{i \mid p_{i}=\min _{i} p_{i}=\underline{p}\right\}, M=\left\{j \mid q_{j}=\max _{j} q_{j}=\bar{q}\right\} .
$$

Pick $i_{0} \in m, j_{0} \in M$. Then $q_{j_{0}}=\left(\sum_{i} p_{i} x_{i} a_{i j_{0}} y_{j_{0}}\right)^{-1} \leqq p_{i_{0}}^{-1}$ and similarly $p_{i_{0}} \geqq q_{j_{0}}^{-1}$, forcing $q_{j_{0}}=p_{i_{0}}^{-1}=\underline{p}^{-1}$. But equality is possible only if $p_{i}=\underline{p}$ when $i \in E_{j_{0}}$. Whence $p_{i}=\underline{p}$ when $i \in E_{j}$ and $j \in M$. Thus $\mathrm{U}_{j \in M} E_{j} \leqq m$ and it follows that $\left.\overline{A(m} \mid M\right]=0$. In the same way $p_{i_{0}}=q_{j_{0}}^{-1}$ is possible only if $q_{j}=\bar{q}$ for all $j \in F_{i_{0}}$. Whence $q_{j}=\bar{q}$ when $j \in F_{i}$ and $i \in m$. Thus $\bigcup_{i \epsilon_{m}} F_{i} \subseteq M$ and it follows that $A[m \mid M)=0$.

On $m \times M, p_{i} q_{j}=\underline{p} \bar{q}=1$ and it follows that $B[m \mid M]=B^{\prime}[m \mid M]$ is doubly stochastic. In particular $m$ and $M$ must have the same size.

If $A$ is fully indecomposable, $A(m \mid M]$ and $A[m \mid M)$ thus cannot exist. In such a case $A=A[m \mid M]$. Thus $D_{1} A D_{2}=D_{1}^{\prime} A D_{2}^{\prime}$, and $D_{1}$ and $D_{2}$ are themselves unique up to a scalar multiple.

If $A(m \mid M]$ and $A[m \mid M)$ exist, $B(m \mid M)$ and $B^{\prime}(m \mid M)$ exist and are each doubly stochastic matrices of order less than $N$. Furthermore $B(m \mid M)=D_{1}^{\prime \prime} A(m \mid M) D_{2}^{\prime \prime} \quad$ and $\quad B^{\prime}(m \mid M)=D_{1}^{\prime \prime \prime} A(m \mid M) D_{2}^{\prime \prime \prime}$ where the $D^{\prime}$ s are diagonal matrices with positive main diagonals. The argument may be repeated on these submatrices until $D_{1} A D_{2}=$ $D_{1}^{\prime} A D_{2}^{\prime}$ is established. Note that $D_{1}$ and $D_{2}$ no longer need be unique up to a scalar multiple. 
The necessity of total support for the existence of $D_{1} A D_{2}$ is an immediate consequence of the celebrated theorem of G. Birkhoff [1] which states that the set of doubly stochastic matrices of order $N$ is the convex hull of the $N \times N$ permutation matrices.

The sufficiency of the condition and the remarks concerning the iteration will follow in part from the following lemmas.

Lemma 1. If $A$ is a row stochastic matrix, and $\beta_{1}, \cdots, \beta_{N}$ are the column sums of $A$, then $\prod_{k=1}^{N} \beta_{k} \leqq 1$, with equality only if each $\beta_{k}=1$.

Proof. Let $A$ have column sums $\beta_{1}, \cdots, \beta_{N}$. Of course, each $\beta_{k} \geqq 0$ and $\sum_{k=1}^{N} \beta_{k}=N$. By the arithmetic-geometric mean inequality

$$
\prod_{k=1}^{N} \beta_{k} \leqq\left[(1 / N) \sum_{k=1}^{N} \beta_{k}\right]^{N}=1
$$

with equality occurring only if each $\beta_{k}=1$.

LEMmA 2. Let $A=\left(a_{i j}\right)$ be an $N \times N$ matrix with total support and suppose that if $1 \leqq i, j \leqq N,\left\{x_{i, n}\right\}$ and $\left\{y_{j, n}\right\}$ are positive sequences such that $x_{i, n} y_{j, n}$ converges to a positive limit for each $i, j$ such that $a_{i j} \neq 0$. Then there exist convergent positive sequences $\left\{x_{i, n}^{\prime}\right\},\left\{y_{j, n}^{\prime}\right\}$ with positive limits such that $x_{i, n}^{\prime} y_{j, n}^{\prime}=x_{i, n} y_{j, n}$ for all $i, j, n$.

Proof. Consider first the case in which $A$ is fully indecomposable. Let $E^{(1)}=\{1\}, F^{(1)}=\left\{j \mid a_{1 j}>0\right\}$, and $E^{(s)}=\left\{i \notin \bigcup_{k=1}^{s-1} E^{(k)} \mid\right.$ for some $\left.j \in F^{(s-1)}, a_{i j}>0\right\}, F^{(s)}=\left\{j \notin \bigcup_{k=1}^{s-1} F^{(k)} \mid\right.$ for some $\left.i \in E^{(s)}, a_{i j}>0\right\}$ when $s>1$. The sets $E^{(s)}$ and $F^{(s)}$ are void for sufficiently large $s$, e.g., for $s>N$. Define $E=\bigcup_{k} E^{(k)}$ and $F=\bigcup_{k} F^{(k)}$. Since $A$ has total support, the first row of $A$ contains a nonzero element; thus $F^{(1)}$ is nonvoid. Since $F^{(1)} \subseteq F, F$ is nonvoid. Also since $\{1\}=E^{(1)} \sqsubseteq E, E$ is nonvoid.

Suppose $E$ is a proper subset of $\{1, \cdots, N\}$. Pick $i \notin E, j \in F$. Then $j \in F^{(s)}$ for some $s$. Since $i \notin E$, certainly $i \notin \bigcup_{k=1}^{s} E^{(k)}$. Certainly then it could not be that $a_{i j}>0$ for then $i \in E^{(s+1)} \subseteq E$, a contradiction. Whence $i \notin E, j \in F \Longrightarrow a_{i j}=0$, i.e., $A(E \mid F]=0$. In the same way it follows that if $F \neq\{1, \cdots, N\}, A[E \mid F)=0$.

Define an $N \times N$ matrix $H=\left(h_{i j}\right)$ as follows. If $a_{i j}=0$, set $h_{i j}=0$. If $a_{i j} \neq 0$ and $a_{i j}$ lies on $t$ positive diagonals in $A$, set $h_{i j}=$ $t / \tau$ where $\tau$ is the total number of positive diagonals in $A$. Then $H$ is doubly stochastic and $h_{i j}=0$ if and only if $a_{i j}=0$. Suppose $E$ contains $u$ elements and $F$ contains $v$ elements. Since $H(E \mid F]=0$, $\sum_{i \in B} \sum_{j \in F} h_{i j}=v$, and since either $F=\{1, \cdots, N\}$ or $H[E \mid F)=0$, $\sum_{i \in \mathbb{E}} \sum_{j \in F} h_{i j}=u$. Thus $E$ and $F$ have the same number of elements. 
But $E$ and $F$ cannot be proper subsets of $\{1, \cdots, N\}$ if $A$ is assumed to be fully indecomposable. Thus $E=F=\{1, \cdots, N\}$.

Define $x_{i, n}^{\prime}=x_{1, n}^{-1} x_{i, n}$ and $y_{j, n}^{\prime}=x_{1, n} y_{j, n}$ for all $i, j, n$. Then $x_{i, n}^{\prime} y_{j, n}^{\prime}=$ $x_{i, n} y_{j, n}$ for all $i, j, n$. Since $x_{1, n}^{\prime}=1$ for all $n$, certainly $x_{1, n}^{\prime} \rightarrow 1$. For $j \in F^{(1)}, y_{j, n}^{\prime}=x_{1, n}^{\prime} y_{j, n}^{\prime}=x_{1, n} y_{j, n}$ has a positive limit.

Inductively suppose that it is known that $x_{i, n}^{\prime}$ and $y_{j, n}^{\prime}$ converge to positive limits when $i \in \bigcup_{k=1}^{s-1} E^{(k)}$ and $j \in \bigcup_{k=1}^{s-1} F^{(k)}$. For $i \in E^{(s)}$ there is a $j_{s-1} \in F^{(s-1)}$ such that $a_{i j_{s-1}}>0$. Thus $x_{i, n}^{\prime}=x_{i, n}^{\prime} y_{j_{s-1}, n}^{\prime} / y_{j_{s-1}, n}^{\prime}=$ $x_{i, n} y_{j_{s-1}, n} / y_{j_{s-1}, n}^{\prime}$ has a positive limit. Then for $j \in F^{(s)}$ there is a $i_{s} \in E^{(s)}$ such that $a_{i_{s} j}>0$. Whence $y_{j, n}^{\prime}=x_{i_{s}, n}^{\prime} y_{j, n}^{\prime} / x_{i_{s}, n}^{\prime}=x_{i_{s}, n} y_{j, n} / x_{i_{s}, n}^{\prime}$ has a positive limit. This completes the proof in case $A$ is fully indecomposable.

If $A$ is not fully indecomposable, then neither is the corresponding doubly stochastic matrix $H$. This means that there exist permutations $P$ and $Q$ such that $P H Q=H_{1} \oplus \cdots \oplus H_{g}$ where each $H_{k}$ is doubly stochastic and fully indecomposable. Thus also $P A Q=A_{1} \oplus \cdots \oplus A_{g}$ where each $A_{k}$ has total support and is fully indecomposable. The above argument may be repeated on each of the $A_{k}$.

Now we return to the theorem. Suppose $A$ has support. Define an iteration on $A$ as follows. where

Let $x_{i, 0} \equiv 1, y_{j, 0} \equiv\left(\sum_{i=1}^{N} \alpha_{i j}\right)^{-1}$ and set $x_{i, n+1}=\alpha_{i, n}^{-1} x_{i, n}, y_{j, n+1}=\beta_{j, n}^{-1} y_{j, n}$

$$
\alpha_{i, n}=\sum_{j=1}^{N} x_{i, n} a_{i j} y_{j, n} ; \quad \beta_{j, n}=\sum_{i=1}^{N} \alpha_{i, n}^{-1} x_{i, n} \alpha_{i j} y_{j, n},
$$

$i=1, \cdots, N, j=1, \cdots, N, n=0,1, \cdots$. Note that $\left(x_{i, n} a_{i j} y_{j, n}\right)$ is column stochastic and $\left(x_{i, n+1} a_{i j} y_{j, n}\right)$ is row stochastic. Then in particular

$$
y_{j, n}=\left(\sum_{i=1}^{N} x_{i, n} a_{i j}\right)^{-1} \leqq x_{i_{0}, n}^{-1} a_{i_{0} j}^{-1} \leqq x_{i_{0}, n}^{-1} a^{-1}
$$

where $i_{0}$ is such that $a_{i_{0} j}>0$ and $a$ is the minimal positive $a_{i j}$. Thus $x_{i, n} y_{j, n} \leqq a^{-1}$ if $\alpha_{i j}>0$.

Let $A$ have a positive diagonal corresponding to a permutation $\sigma$, and set $s_{n}=\prod_{i=1}^{N} x_{i, n} y_{\sigma(i), n}$ and $s_{n}^{\prime}=\prod_{i=1}^{N} x_{i, n+1} y_{\sigma(i), n}$. By Lemma 1 and the preceding remark, $s_{n} \leqq s_{n}^{\prime} \leqq s_{n+1} \leqq a^{-N}$. Thus $s_{n} \rightarrow L$ and $s_{n}^{\prime} \rightarrow L$ where $0<L \leqq a^{-N}$. Whence $\prod_{j=1}^{N} \beta_{j, n}=s_{n}^{\prime} / s_{n+1} \rightarrow 1$. This is impossible unless each $\beta_{j, n} \rightarrow 1$ since $\prod_{k=1}^{N} \beta_{k}$ has a unique maximal value of 1 only when $\beta_{1}=\cdots=\beta_{N}=1$. Similarly each $\alpha_{i, n} \rightarrow 1$.

Thus if $A$ has a positive diagonal, the limit points of the sequence of matrices generated by the iteration are doubly stochastic. However, two such limit points are diagonally equivalent. Suppose that $A_{n}$ is the $n$th matrix in the iteration and that $A_{n_{k}} \rightarrow B$ and $A_{m_{k}} \rightarrow C$. Observe that for any given pair $i, j b_{i j} \neq 0 \Leftrightarrow c_{i j} \neq 0$. For any per- 
mutation $\sigma, \prod_{i=1}^{N} b_{i, \sigma(i)}=\prod_{i=1}^{N} c_{i, \sigma(i)}=L \prod_{i=1}^{N} a_{i, \sigma(i)}$. Then certainly $b_{i j} \neq$ $0 \Longrightarrow c_{i j} \neq 0$, for suppose $b_{i_{0} j_{0}} \neq 0$. Then $b_{i_{0} j_{0}}$ lies on a positive diagonal. The corresponding diagonal in $C$ would have a positive product. Thus $\mathrm{c}_{i_{0} j_{0}} \neq 0$. In the same way $c_{i j} \neq 0 \Rightarrow b_{i j} \neq 0$. If in addition $A$ has total support then $a_{i j} \neq 0 \Leftrightarrow b_{i j} \neq 0 \Leftrightarrow c_{i j} \neq 0$.

By construction there exist matrices $\widetilde{D}_{1, k}=\operatorname{diag}\left(w_{1, k}, \cdots, w_{N, k}\right)$ and $\widetilde{D}_{2, k}=\operatorname{diag}\left(z_{1, k}, \cdots, z_{N, k}\right)$ with positive main diagonals such that $A_{m_{k}}=\widetilde{D}_{1, k} A_{n_{k}} \widetilde{D}_{2, k}$. For $b_{i j}>0, w_{i, k} z_{j, k} \rightarrow c_{i j} b_{i j}^{-1}$. By Lemma 2 there exist positive sequences $\left\{w_{i, k}^{\prime}\right\}$ and $\left\{z_{j, k}^{\prime}\right\}$ converging to positive limits such that $w_{i, k}^{\prime} w_{j, k}^{\prime}=w_{i, l, k} z_{j, k}$, for all $i, j, k$. If

$$
D_{1}=\lim _{k \rightarrow \infty} \operatorname{diag}\left(w_{1, k}^{\prime}, \cdots, w_{N, k}^{\prime}\right) \text { and } D_{2}=\lim _{k \rightarrow \infty} \operatorname{diag}\left(z_{1, k}^{\prime}, \cdots, z_{N, k}^{\prime}\right) \text {, }
$$

then $C=D_{1} B D_{2}$. By the uniqueness part of the theorem, $B=C$. It follows that the iteration converges. It is clear from Birkhoff's theorem that no limit to the iteration is possible without at least one positive diagonal.

Suppose $A$ has total support. Let $D_{1, n}=\operatorname{diag}\left(x_{1, n}, \cdots, x_{N, n}\right)$ and $D_{2, n}=\operatorname{diag}\left(y_{1, n}, \cdots, y_{N, n}\right)$. Then $B=\lim _{n \rightarrow \infty} D_{1, n} A D_{2, n}$ exists and $b_{i j} \neq$ $0 \Leftrightarrow a_{i j} \neq 0$. When $a_{i j}>0, x_{i, n} y_{j, n} \rightarrow b_{i j} a_{i j}^{-1}$. By Lemma 2 there are convergent positive sequences $\left\{x_{i, n}^{\prime}\right\},\left\{y_{j, n}^{\prime}\right\}$ with positive limits such that $x_{i, n}^{\prime} y_{j, n}^{\prime}=x_{i, n} y_{j, n}$ for all $i, j, n$. Let $D_{1}=\lim _{n \rightarrow \infty} \operatorname{diag}\left(x_{1, n}^{\prime}, \cdots, x_{N, n}^{\prime}\right)$ and $D_{2}=\lim _{n \rightarrow \infty} \operatorname{diag}\left(y_{1, n}^{\prime}, \cdots, y_{N, n}^{\prime}\right)$. Then $B=D_{1} A D_{i}$.

Finally we observe that if $A$ has support which is not total, then by Birkhoff's theorem, there is a nonzero element of $A$ which tends to zero in the iteration. In fact every nonzero element of $A$ which is not on a positive diagonal must do so. If the limit matrix could be put in the form $D_{1} A D_{2}$ then some term $x_{i} a_{i j} y_{j}=0$ where $a_{i j}>0$. But then either $x_{i}=0$ or $y_{j}=0$. The former leads to a row of zeros and the latter to a column of zeros in $D_{1} A D_{2}$. In either case $D_{1} A D_{2}$ could not be doubly stochastic.

The authors wish to express their gratitude to Professor Albert Newhouse for his help on numerical examples connected with this problem. They also are indebted to Professors M. Marcus and H. Schneider and the referee for suggestions which simplified some of the proofs.

\section{REFERENCES}

1. G. Birkhoff, Tres observaciones sobre el algebra lineal, Univ. Nac. Tucumán Rev. Ser. (A) 5 (1946), 147-150.

2. R. Brualdi, S. Parter and H. Schneider, The diagonal equivalence of a nonnegative matrix to a stochastic matrix (to appear).

3. M. Marcus and H. Minc, A survey of matrix theory and matrix inequalities, Allyn 
and Bacon, Boston, 1964.

4. M. Marcus and M. Newman, unpublished paper.

5. J. Maxfield and H. Minc, A doubly stochastic matrix equivalent to a given matrix, Notices Amer. Math. Soc. 9 (1962), 309.

6. R. Sinkhorn, A relationship between arbitrary positive matrices and doubly stochastic matrices, Ann. Math. Statist. 35 (1964), 876-879.

Received February 20, 1966.

UNIVERSITY OF HOUSTON

Houston, TeXas 


\section{PACIFIC JOURNAL OF MATHEMATICS}

\section{EDITORS}

H. SAMELSON

Stanford University

Stanford, California

J. P. JANS

University of Washington

Seattle, Washington 98105

\section{J. DugunduI}

University of Southern California Los Angeles, California 90007

RICHARD ARENS

University of California

Los Angeles, California 90024

\section{ASSOCIATE EDITORS}
E. F. BECKENBACH
B. H. NEUMANN
F. WOLF
K. YoSIDA

\section{SUPPORTING INSTITUTIONS}

\author{
UNIVERSITY OF BRITISH COLUMRIA \\ CALIFORNIA INSTITUTE OF TECHNOLOGY \\ UNIVERSITY OF CALIFORNIA \\ MONTANA STATE UNIVERSITY \\ UNIVERSITY OF NEVADA \\ NEW MEXICO STATE UNIVERSITY \\ OREGON STATE UNIVERSITY \\ UNIVERSITY OF OREGON \\ OSAKA UNIVERSITY \\ UNIVERSITY OF SOUTHERN CALIFORNIA
}

\author{
STANFORD UNIVERSITY \\ UNIVERSITY OF TOKYO \\ UNIVERSITY OF UTAH \\ WASHINGTON STATE UNIVERSITY \\ UNIVERSITY OF WASHINGTON \\ AMERICAN MATHEMATICAL SOCIETY \\ CHEVRON RESEARCH CORPORATION \\ TRW SYSTEMS \\ NAVAL ORDNANCE TEST STATION
}

Mathematical papers intended for publication in the Pacific Journal of Mathematics should be typewritten (double spaced). The first paragraph or two must be capable of being used separately as a synopsis of the entire paper. It should not contain references to the bibliography. Manuscripts may be sent to any one of the four editors. All other communications to the editors should be addressed to the managing editor, Richard Arens at the University of California, Los Angeles, California 90024 .

50 reprints per author of each article are furnished free of charge; additional copies may be obtained at cost in multiples of 50 .

The Pacific Journal of Mathematics is published monthly. Effective with Volume 16 the price per volume (3 numbers) is $\$ 8.00$; single issues, $\$ 3.00$. Special price for current issues to individual faculty members of supporting institutions and to individual members of the American Mathematical Society: $\$ 4.00$ per volume; single issues $\$ 1.50$. Back numbers are available.

Subscriptions, orders for back numbers, and changes of address should be sent to Pacific Journal of Mathematics, 103 Highland Boulevard, Berkeley 8, California.

Printed at Kokusai Bunken Insatsusha (International Academic Printing Co., Ltd.), No. 6, 2-chome, Fujimi-cho, Chiyoda-ku, Tokyo, Japan.

\section{PUBLISHED BY PACIFIC JOURNAL OF MATHEMATICS, A NON-PROFIT CORPORATION}

The Supporting Institutions listed above contribute to the cost of publication of this Journal, but they are not owners or publishers and have no responsibility for its content or policies. 


\section{Pacific Journal of Mathematics}

\section{Vol. 21, No. 2 December, 1967}

Arne P. Baartz, The measure algebra of a locally compact semigroup ..... 199

Robert F. Brown, On maps with identical fixed point sets............. 215

C. Buttin, Existence of a homotopy operator for Spencer's sequence in the analytic case ..................................... 219

Henry Werner Davis, An elementary proof that Haar measurable almost periodic functions are continuous ........................ 241

Zeev Ditzian, On asymptotic estimates for kernels of convolution transforms ...................................... 249

Robert E. Edwards, Boundedness principles and Fourier theory ......... 255

John A. Hildebrant, On compact unithetic semigroups ............... 265

Marinus A. Kaashoek and David Clark Lay, On operators whose Fredholm set is the complex plane ............................ 275

Sadao Kató, Canonical domains in several complex variables ........... 279

David Clifford Kay, The ptolemaic inequality in Hilbert geometries.... . . . 293

Joseph D. E. Konhauser, Biorthogonal polynomials suggested by the Laguerre polynomials ............................. 303

Kevin Mor McCrimmon, Macdonald's theorem with inverses .......... 315

Harry Eldon Pickett, Homomorphisms and subalgebras of multialgebras .................................... 327

Richard Dennis Sinkhorn and Paul Joseph Knopp, Concerning nonnegative matrices and doubly stochastic matrices ..............

Erling Stormer, On anti-automorphisms of von Neumann algebras ...

Miyuki Yamada, Regular semi-groups whose idempotents satisfy permutation identities .......................... 